\title{
Real-World Implications of Changing Blood Pressure Targets in Urban Primary Care
}

\author{
Shari D. Bolen, MD, MPH', 2,3,4, Douglas Einstadter, MD, MPH',2,3,4, Thomas E. Love, PhD 1,2,3,4, \\ Scott Husak, $B S^{1,2,4}$, Jonathan Lever, MPH, NRP², and Randall D. Cebul, MD ${ }^{1,2,3,4}$ \\ ${ }^{1}$ Center for Health Care Research and Policy, Case Western Reserve University at The MetroHealth Medical Center, Cleveland, OH, USA; ${ }^{2}$ Better \\ Health Partnership, Cleveland, OH, USA; ${ }^{3}$ Department of Population and Quantitative Health Sciences, Case Western Reserve University, \\ Cleveland, OH, USA; ${ }^{4}$ Department of Medicine, The MetroHealth Medical Center, Cleveland, OH, USA.
}

KEY WORDS: hypertension; primary care; quality of care.

$\mathrm{J}$ Gen Intern Med 33(12):2027-9

DOI: $10.1007 / \mathrm{s} 11606-018-4595-3$

(C) Society of General Internal Medicine 2018

\section{INTRODUCTION}

The impact of the new ACC/AHA hypertension guideline ${ }^{1}$ in primary care is uncertain. We describe implications of the guideline for: (1) patients with diagnosed hypertension in a diverse regional health improvement collaborative (RHIC) and (2) patients without diagnosed hypertension at one safety-net health system within the RHIC.

\section{METHODS}

We used data from Better Health Partnership (BHP), a Northeast Ohio RHIC that includes 80 primary care clinics across nine health systems. ${ }^{2}$ BHP member clinics use electronic health record data to report blood pressure (BP) results twice yearly for patients with hypertension using the last BP in the reporting period. In the full sample of hypertensive patients, BHP measures blood pressure control in two ways: (1) BP < $140 / 90 \mathrm{mmHg}$ for most, and $\mathrm{BP}<150 / 90$ for those over age 60 without diabetes (based on Joint National Committee (JNC) 8 guidelines) $)^{3}$ and (2) $\mathrm{BP}<140 / 90 \mathrm{mmHg}$ for all patients (based on JNC 7). ${ }^{4}$ In the second sample of patients without hypertension, we identified all patients with $\geq 2$ visits over a 2 -year period at any of 20 primary care clinics at MetroHealth, a safety-net system in Cleveland that also participates in BHP. For those without a hypertension diagnosis, we determined how many would be diagnosed with hypertension using a $\mathrm{BP}$ reading of $\geq 130 / 80 \mathrm{mmHg}$ measured at two consecutive primary care visits. We ascertained whether medications would be recommended for newly diagnosed patients based on the presence of diabetes, chronic kidney disease or a 10 -year cardiovascular risk score $\geq 10 \%$ using the pooled cohort equation. ${ }^{5}$ If the risk score could not be calculated, we assumed a low $(<10 \%)$ risk.

Published online July 31, 2018

\section{RESULTS}

There were 194,742 hypertensive patients seen by 813 providers at 80 primary care clinics between July 1, 2016 and June 30, 2017. Clinic-level characteristics range from 0 to $63 \%$ Medicaid patients, 0 to $98 \%$ black patients, and 0 to $48 \%$ patients who prefer speaking Spanish. In this sample, $84 \%$ received at least one BP medication, $61 \%$ received at least two BP medications, and $39 \%$ received three or more BP medications. Using the current BHP BP targets, 79\% and 74\% had good BP control using the JNC8 and JNC7 guidelines, respectively (Fig. 1). Using the ACC/AHA guideline BP control target $(\mathrm{BP}<130 / 80 \mathrm{mmHg}$ ), the proportion with good BP control would decrease to $35 \%$ (Fig. 1). In the second sample, there were 57,818 of 107,921 patients without an established diagnosis of hypertension. Patients seen at MetroHealth are diverse, with 30\% Medicaid, 7\% uninsured, $30 \%$ Medicare, $33 \%$ commercial insurance, $52 \%$ white, $37 \%$ black, $5 \%$, and a median household income of $\$ 40,099$. Based on the ACC/AHA guideline, 19,776 new patients would be diagnosed with hypertension, increasing the overall prevalence of hypertension by $19 \%$, from $46 \%(n=50,103)$ to $65 \%(n=69,879)$ (Fig. 2). In addition, 5129 newly diagnosed patients with hypertension would receive a recommendation to start medication, increasing the proportion of total primary care patients $(N=107,921)$ on BP medications by $4.7 \%$ (from 40.9 to $45.7 \%)$.

\section{DISCUSSION}

Our study is the first to use real-world blood pressure measurement when considering implications of the new $\mathrm{ACC} /$ AHA hypertension guideline. Adoption of the ACC/AHA hypertension guideline would meaningfully increase the number of persons identified with hypertension, decrease the percentage of hypertension patients under good BP control, and add significant medication burden. While these findings are not surprising, it is important to recognize the magnitude of potential changes when deciding on quality targets. Our regional results demonstrate a higher percentage of patients who would be identified as hypertensive and a higher percentage 


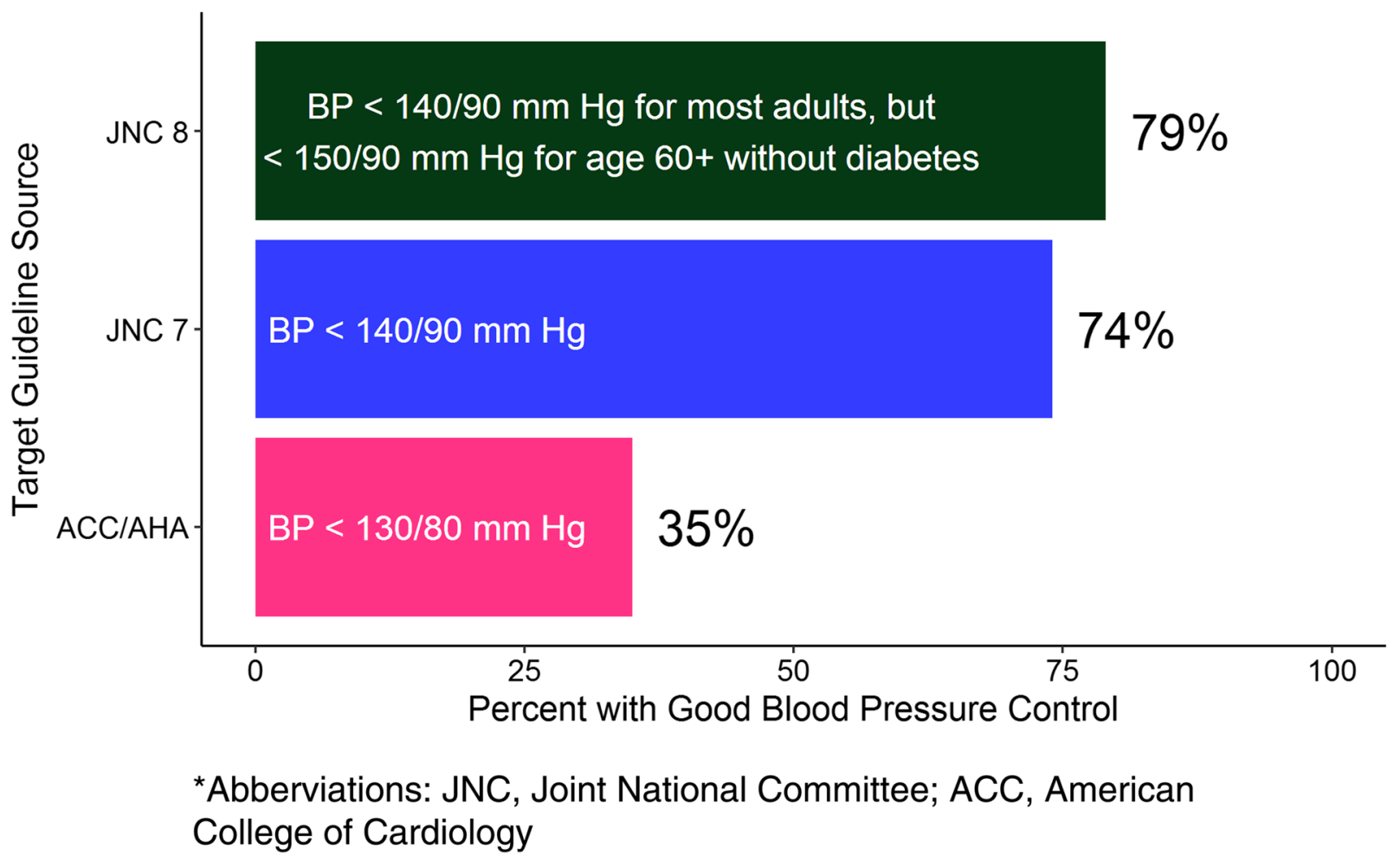

Fig. 1 Percent of hypertensive adults under good blood pressure control using different blood pressure targets within Better Health Partnership in 2016-2017 ( $N=194,742$ hypertensive adults). *Abbreviations: JNC, Joint National Commission; ACC, American College of Cardiology

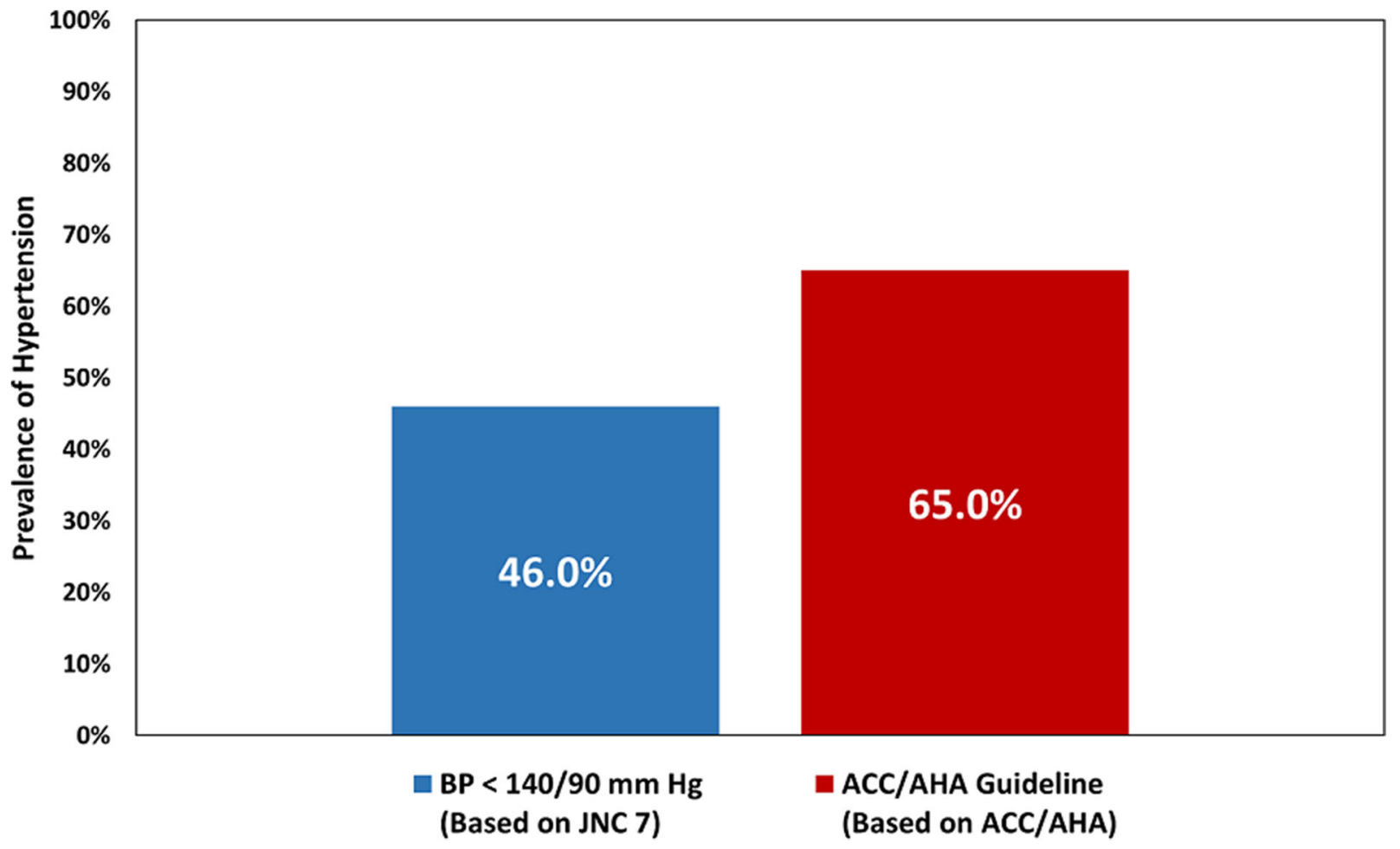

*Abberviations: JNC, Joint National Committee; ACC, American College of Cardiology; AHA, American Heart Association

Fig. 2 Prevalence of HTN under various hypertension guidelines for primary care patients at The MetroHealth System $(N=107,921)$. *Abbreviations: JNC, Joint National Commission; ACC, American College of Cardiology; AHA, American Heart Association 
who would be recommended a BP medication compared with national estimates from the recently reported National Health and Nutrition Examination Survey (NHANES) data. ${ }^{6}$ Using NHANES, Munter et al. reported an additional $13.7 \%$ of people diagnosed with hypertension and an additional 1.9\% who would require medication treatment based on the ACC/ AHA guideline versus the additional 19 and $4.7 \%$ we found respectively in our region. ${ }^{6}$ The differences between our results may be due to a variety of factors including differences in patient populations (regional versus national) or in methods for BP measurement (real world versus study protocol) between the two studies. We encourage others to review their data to better determine local policy and clinical implications of instituting different quality targets. We also encourage future research on the effect of the new guideline on provider engagement, patient adherence, patient quality of life, and clinical outcomes.

Acknowledgments: No external funding supported the current work for this manuscript. Dr. Bolen, Dr. Einstadter, and Mr. Lever's time is supported by a CDC funded REACH (Racial and Ethnic Approaches to Community Health) grant and by grants from the Ohio Department of Medicaid to work on improving the cardiovascular health of Medicaid enrollees. Drs. Love and Cebul are also supported on related grants from the Ohio Department of Medicaid. Dr. Love and Mr. Husak time is supported by Better Health Partnership - a nonprofit regional health improvement collaborative.
Corresponding Author: Shari D. Bolen, MD, MPH; Center for Health Care Research and Policy Case Western Reserve University at The MetroHealth Medical Center, Cleveland, OH, USA (e-mail: sdb73@case.edu).

Compliance with ethical standards:

Conflicts of interest: The authors declare that they have no conflict of interest.

\section{REFERENCES}

1. Whelton PK, Carey RM, Aronow WS, et al. 2017 ACC/AHA/AAPA/ABC/ ACPM/AGS/APhA/ASH/ASPC/NMA/PCNA Guideline for the prevention, detection, evaluation, and management of high blood pressure in adults: a report of the American College of Cardiology/American Heart Association Task Force on clinical practice guidelines. Hypertension (Dallas, Tex. : 1979). 2017.

2. Better Health Partnership. Transforming health care together. Available at: http://www.betterhealthpartnership.org/. Accessed 06/09/2018.

3. James PA, Oparil S, Carter BL, et al. 2014 Evidence-based guideline for the management of high blood pressure in adults: report from the panel members appointed to the Eighth Joint National Committee (JNC 8). Jama. 2014;311(5):507-520.

4. Chobanian AV, Bakris GL, Black HR, et al. Seventh report of the Joint National Committee on prevention, detection, evaluation, and treatment of high blood pressure. Hypertension (Dallas, Tex. : 1979). 2003;42(6):1206-1252.

5. Stone NJ, Robinson JG, Lichtenstein AH, et al. 2013 ACC/AHA guideline on the treatment of blood cholesterol to reduce atherosclerotic cardiovascular risk in adults: a report of the American College of Cardiology/American Heart Association Task Force on practice guidelines. Circulation. 2014;129(25 Suppl 2):S1-45.

6. Muntner P, Carey RM, Gidding S, et al. Potential US population impact of the 2017 ACC/AHA high blood pressure guideline. Circulation. 2018;137(2):109-118 